\title{
Erosions, dépots et transits sédimentaires associés dans le bassin de Marennes-Oléron
}

\author{
Pierre Le Hir ${ }^{1, *}$, Stéphane Kervella ${ }^{2}$, Patrice Walker ${ }^{3}$ et Isabelle Brenon ${ }^{4}$ \\ ${ }^{1}$ Ifremer, laboratoire Dyneco/Physed, centre Ifremer de Brest, B.P. 70, 29280 Plouzané, France \\ 2 Ifremer LERPC Place Gaby Coll, B.P. 5, 17137 L'Houmeau, France \\ ${ }^{3}$ Créocéan, zone Technocean, Chef de Baie, 17000 La Rochelle, France \\ ${ }^{4}$ Université de La Rochelle, UMR LIENSS, 2 rue Olympe De Gouges, 17000 La Rochelle, France \\ *: Corresponding author : Pierre Le Hir, Tél : +33(0)2 982243 40, email address : \\ pierre.le.hir@ifremer.fr
}

\section{Résumé}

La gestion des sédiments dans le bassin de Marennes-Oléron nécessite une connaissance des flux particulaires et des bilans sédimentaires à l'échelle du bassin, et une description des conditions dominantes dans lesquelles les transits se produisent. Un modèle de dynamique sédimentaire prenant en compte le transport simultané des sables et des vases sous l'effet des vagues et des courants a été appliqué au secteur côtier des Pertuis Charentais. La consolidation des vases et des mélanges de sable et de vase est simulée. La validation est réalisée à l'aide de mesures de courant, de suivis altimétriques en 8 points pendant un an, d'enregistrements de pression pour les vagues sur estran et de mesures de turbidité.

Une dissymétrie entre l'est et l'ouest du bassin pour la turbidité est constatée, en accord avec une circulation résiduelle nord/sud. Cette circulation moyenne générée par la marée entraîne un transit résiduel de matériel fin vers le sud, qui est partiellement piégé dans le bassin. Le modèle identifie la zone à l'est du pertuis d'Antioche comme principale source de cette dérive, ce qui reste à valider. Les tempêtes sont susceptibles d'inverser ces flux, mais sur des périodes courtes. Pourtant, ce sont elles et les agitations locales qui génèrent les fortes turbidités. Le modèle met en évidence la variabilité de la nature du sédiment superficiel, à l'échelle de la marée ou à la suite de tempêtes.

\begin{abstract}
Managing sediments in the baie de Marennes-Oléron (Atlantic coast of France) can be achieved providing particulate fluxes at the scale of the basin are available, as well as tidal and meteorological conditions during which major transports occur. A sediment dynamics model accounting for tide- and wave-induced sand and mud transport has been applied to the Pertuis Charentais area. Consolidation of pure mud and mixtures of sand and mud is accounted for. The model validation is achieved by current and turbidity measurements, local altimetric surveys during one year and pressure measurements for waves on the tidal flat.
\end{abstract}

An asymmetry between eastern and western halves of the bay is observed on turbidity signals, in agreement with a residual circulation from north to south. This flow generates a net fine sediment 
transport towards the south. A part of it is deposited within the bay. According to the model, the eroded area east of Antioche inlet would be the main source of this southward flux: this feature has to be validated. The flux can be inversed during storms, but for a short duration. However, waves are dominant in the resuspension process. Last, the variability of sediment nature is demonstrated with the model, at different time scales in relation with tidal phases and storm.

\section{Mots clefs}

Dynamique, sédiment, sable, vase, érosion, dépôt, consolidation, flux particulaire, modélisation, Marennes 


\section{INTRODUCTION}

La gestion des sédiments dans un domaine côtier nécessite de connaître les sources et puits de sable et de vase, qui peuvent résulter des flux au large mais aussi des érosions et des dépôts, ainsi que les cheminements préférentiels des matériaux transportés en suspension ou par charriage près du fond. Par exemple, l'opportunité de draguer un banc sableux venant barrer un chenal de navigation peut être mise en regard d'un besoin de rechargement de plage à proximité. D'éventuelles opérations de dragage par simple remise en suspension des vases doivent faire l'objet d'une étude de dispersion pour optimiser les heures de dragage. L'implantation d'installations conchylicoles susceptibles de provoquer des envasements, ou d'être victimes de brusques ensablements mérite d'être étudiée en fonction des transits de sédiments alentour. Pour faire face à ce type de questions, le Conseil Général de Charente Maritime a coordonné et financé une « étude pour une gestion dynamique des sédiments sur les côtes du bassin de MarennesOléron ».

La connaissance des conditions dans lesquelles se font les transports majeurs est également essentielle, par exemple pour définir les spécifications d'une étude de détail d'un aménagement, ou pour rationaliser les périodes d'entretien annuels. Est-ce que les tempêtes exceptionnelles ont un rôle dominant ? - ou dans certains cas les marées de vive eau ? Se passe-t-il quelque chose pendant les périodes calmes en morte eau, qui justifie d'en tenir compte (consolidation éventuelle) ? Dans le cas du bassin de Marennes-Oléron, les débits de la Charente ont-ils un effet sur les processus sédimentaires en baie ? Ce genre de question peut être abordé à une échelle régionale, en cherchant à décrire et comprendre les mécanismes essentiels.

On sait par ailleurs que la nature sédimentaire du fond conditionne les habitats benthiques, et donc l'implantation et le développement des biocénoses. La composition du sédiment superficiel est susceptible d'évoluer, selon plusieurs échelles de temps : marée, saison, dérive pluri-annuelle, ou encore variation événementielle. Autant que les possibles changements brusques induits par les épisodes de tempête, ce sont aussi les temps de « récupération » du système (on peut parler de résilience), et sa capacité à revenir ou non à une configuration antérieure qui intéressent les environnementalistes du littoral.

Enfin la connaissance de la turbidité elle-même est essentielle, car elle conditionne la pénétration de la lumière, et donc la production phytoplanctonique, qui constitue la principale nourriture des bivalves cultivés. Le cas du bassin de Marennes-oléron (B.MO) est évidemment exemplaire, puisqu'il s'agit du premier bassin ostréicole français.

Pour ces différentes raisons, une étude générale des transports de sédiments dans le B.MO a été menée, sur la base d'un modèle numérique ayant fait l'objet d'une validation soignée. Les principaux résultats sont présentés ci-après. On trouvera successivement une description sommaire du site d'étude et du modèle utilisé (sections II et III), puis quelques résultats de validation (section IV) avant une synthèse sur les mouvements de sédiments modélisés (section V), décrivant d'abord les matières en suspension (MES), puis les flux sédimentaires et les érosions/dépôts qui en résultent, et enfin les évolutions à court terme de la nature du sédiment superficiel.

\section{LE BASSIN DE MARENNES-OLERON}

Situé entre l'île d'Oléron et le littoral atlantique, le bassin de Marennes Oléron (fig.1) est une mer semi-fermée reliée au golfe de Gascogne par le pertuis d'Antioche (large et profond) et le pertuis de Maumusson au sud (étroit, et très dynamique). La moitié de la baie est occupée par de larges estrans, vaseux à l'Est et sablo-vaseux à l'Ouest. L'amplitude de marée atteint $6 \mathrm{~m}$ en vive eau. La marée se propageant depuis les pertuis, les courants de flot convergent vers le centre du bassin à certains moments de la marée, mais une circulation résiduelle des masses d'eau du Nord vers le Sud a été montrée. Entre les îles d'Aix et d'Oléron, ainsi que dans le bassin sud (i.e. au sud du Chapus), de nombreux bancs sableux séparent d'étroits chenaux et manifestent une dynamique intense [1]. L'estuaire de la Charente, long d'environ $50 \mathrm{~km}$ en aval du barrage de StSavinien, a son embouchure à l'Est du bassin. Un bouchon vaseux est observé dans l'estuaire. Le flux particulaire net à la sortie de la Charente est très mal connu, et l'envasement des rives de 
l'estuaire soulève aujourd'hui de gros problèmes d'exploitation portuaire. Les installations conchylicoles occupent schématiquement l'ensemble du tiers inférieur des estrans.

\section{PRESENTATION SUCCINCTE DE LA METHODOLOGIE}

\section{III.1 Modélisation}

Le modèle hydrodynamique bidimensionnel SiAM-2DH [2] est utilisé pour simuler la propagation de la marée et les circulations induites par le vent. II résout les équations de St-Venant en différences finies selon une technique ADI. Les limites du domaine de calcul sont représentées sur la figure 1, ainsi que le maillage, cartésien irrégulier. Dans le bassin, les mailles sont des carrés de $200 \mathrm{~m}$ de coté. La géométrie de la Charente est schématisée par un canal (non visible sur la figure 1) de largeur et profondeur variable de façon à respecter les sections d'écoulement à mi-marée. Les conditions aux limites de marée sont une composition harmonique interpolée le long de la limite marine en fonction de la cartographie de 143 ondes proposée par le SHOM [3]. Le vent mesuré au sémaphore de Chassiron (Nord d'Oléron) est utilisé comme forçage uniforme de la masse d'eau.

Le calcul des vagues a été réalisé à l'aide du modèle SWAN pour une sélection de niveaux d'eau et de conditions de vent, parmi les plus fréquentes dans le secteur d'étude [4]. Pour une simulation réaliste, les vagues sont obtenues par interpolation sur la direction et/ou l'intensité du vent et sur le niveau de marée supposé uniforme dans le plan d'eau. Les houles du large sont sommairement prises en compte, en utilisant la cartographie des vagues calculées pour une tempête d'ouest, corrigée d'un facteur permettant de retrouver les vagues observées à la bouée Gascogne de Méteo-France.
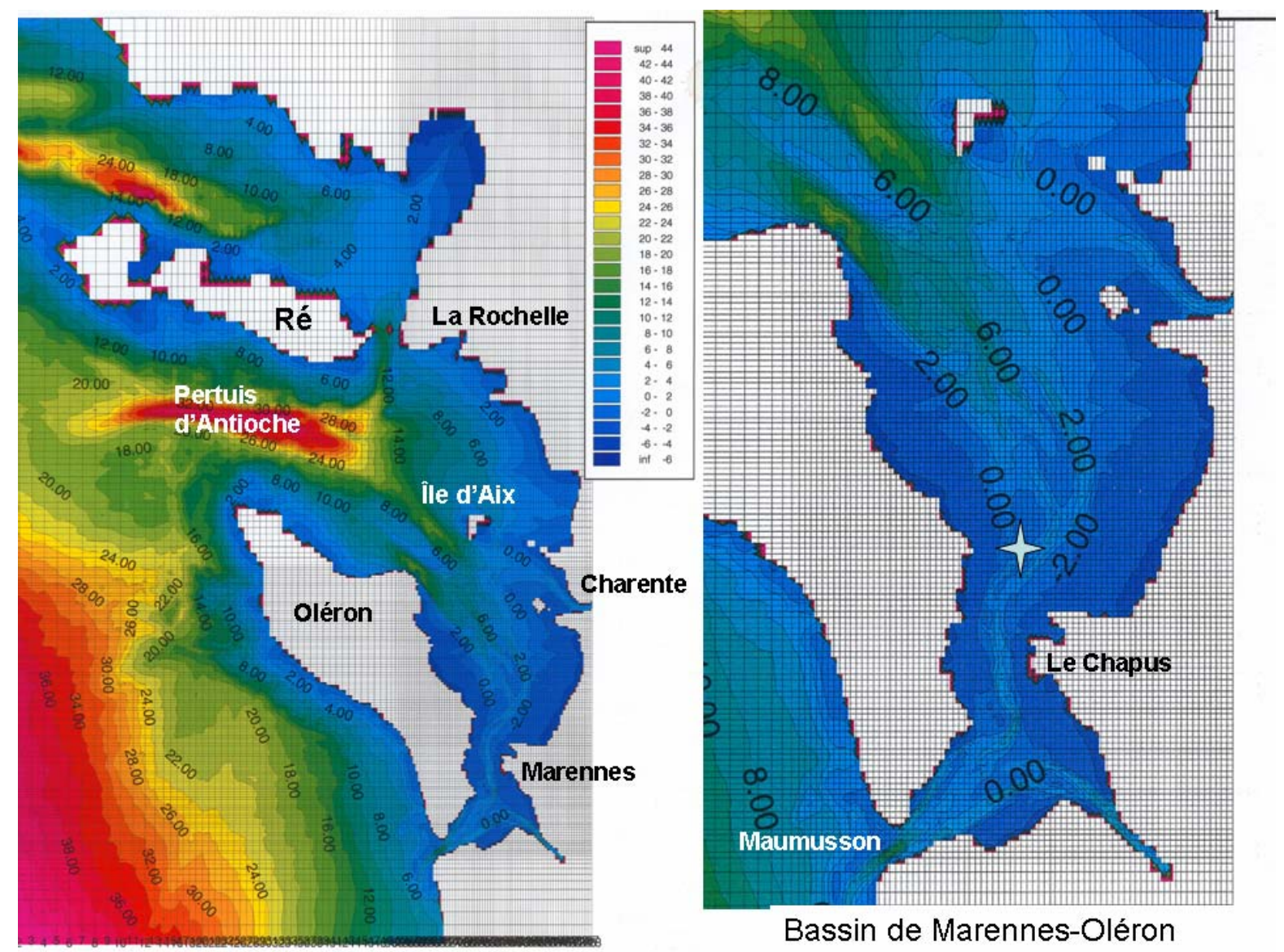

Figure 1: situation du bassin de Marennes-Oléron; présentation du maillage et de la bathymétrie, telle que perçue par le modèle

Le modèle sédimentaire a fait l'objet d'un développement original [5]. Plusieurs classes de sédiment sont considérées, regroupées en 3 types : les «graviers », éventuellement transportés 
par charriage, les sables fins et moyens, transportés en suspension, et les vases également transportées en suspension. Selon la proportion de vase, le sédiment a un comportement cohésif ou non cohésif, caractérisé par une loi d'érosion différente. Si le sédiment est cohésif, l'érodabilité dépend de la concentration relative de la vase, i.e. sa concentration dans l'espace non occupé par les grains de sable. Une attention particulière a été portée aux conditions de dépôt : d'une manière générale, les dépôts sableux sont pris en compte les premiers, et les particules plus fines viennent combler les interstices jusqu'à l'obtention d'une porosité minimale, puis une nouvelle couche de sédiment est constituée. D'autre part, un dépôt vaseux ne sera mélangé au sédiment existant que si celui-ci n'est pas encore consolidé. La consolidation est simulée en résolvant une équation de Gibson pour chaque classe de sédiment. La perméabilité est fonction du diamètre représentatif des petites particules, tandis que la contrainte effective est exprimée en fonction de la concentration relative de vase.

Cinq classes de sédiment ont été retenues (graviers de $2 \mathrm{~mm}$, sables moyens de $300 \mu$ et de $120 \mu$ ), et deux classes de vase, l'une étant utilisée pour « marquer » les vases de Charente. La cartographie des facies sédimentaires du SHOM [6] a été utilisée pour l'initialisation de la distribution des différentes classes de particules.

\section{III.2 Mesures in situ}

Pour la validation du modèle on disposait de mesures de courant par profileur ADCP réalisées par Ifremer dans le cadre du réseau REPER de la région Poitou-Charente, de mesures de MES réalisées selon un échantillonnage temporel très limité, et de deux ou trois enregistrements de sonde de turbidité d'une dizaine de jours. Afin de mieux appréhender la variabilité de la turbidité à différentes échelles, et de caractériser les érosions-dépôts en fonction des forçages, une campagne de mesures a été réalisée d'avril 2007 à avril 2008.

Des sondes multiparamètres (température, salinité, turbidité) ont été placées en 3 points, en bas des estrans Est et Ouest. Des altimètres ALTUS [7] ont été déployés en 8 stations réparties sur l'estran du bassin [8]. Tous ces instruments étaient destinés à mesurer les érosions/dépôts selon un pas de temps de 20' avec une précision millimétrique. Ils disposaient également d'un capteur de pression donnant la hauteur de marée et les surcotes, mais aussi les spectres de vagues (grâce à l'acquisition de salves de 5 minutes à $2 \mathrm{~Hz}$ ): ainsi une base de données de validation des vagues dans le bassin a été créée.

\section{CALIBRATION ET VALIDATION DU MODELE}

La validation du modèle suit une progression par étapes. Après une phase d'ajustement de la rugosité sur le fond, dépendante de la nature sédimentaire, l'hydrodynamique a été validée en comparant courants mesurés et observés pendant plusieurs marées en 4 points représentatifs de la baie. L'accord est très bon, cependant le niveau de basse mer simulé est plus haut d'une vingtaine de $\mathrm{cm}$, parce que le découvrement dans le modèle est légèrement ralenti : ce peut être dû à la non prise en compte dans les mailles de $200 \mathrm{~m}$ des seillons et petits chenaux qui traversent l'estran (en particulier dans la vasière de Brouage) et permettent une évacuation plus rapide de l'eau (cf. une anomalie similaire dans un modèle de la mer de Wadden [9]).

La simulation des vagues n'a pas fait l'objet de calibration, car les mesures ont été effectuées après. La validation a porté sur l'estimation de la contrainte de cisaillement, qui suppose une hypothèse sur la rugosité de peau prise en compte. Le rôle essentiel des vagues est confirmé, mais l'accord entre les résultats de la modélisation et l'ensemble des observations est jugé moyen. La simulation des vagues en mode instationnaire respectant l'évolution réelle du vent mériterait d'être testée.

Certains processus sédimentaires ont été calibrés. Ainsi la consolidation des vases a été paramétrée à l'aide de culots de tassement réalisés par Sogreah en 1995 [10]. Le paramétrage de la vitesse de chute des sédiments cohésifs et des lois d'érosion a été ajusté pour respecter approximativement les variations de concentrations en MES, à la fois dans les chenaux et sur la vasière de Brouage, tout en évitant une dérive du modèle dans le temps, soit en termes de concentration moyenne, soit en termes d'érosion/dépôt. 
La validation des transports sédimentaires s'appuie sur les récents enregistrements de turbidité et d'érosion/dépôt (non montrés ici). A cette fin, la période du $1^{\text {er }}$ mars 2007 au 6 avril 2008 a été simulée, avec des forçages réalistes. La figure 2 illustre le type de comparaison que l'on obtient entre le modèle et la mesure. On observe que les resuspensions par les tempêtes peuvent être très importantes. Leur sous-estimation par le modèle le 5 mars 2008 est plutôt à attribuer à une sous-estimation des vagues ce jour-là, mais, dans l'ensemble, la dynamique des turbidités observées est respectée.

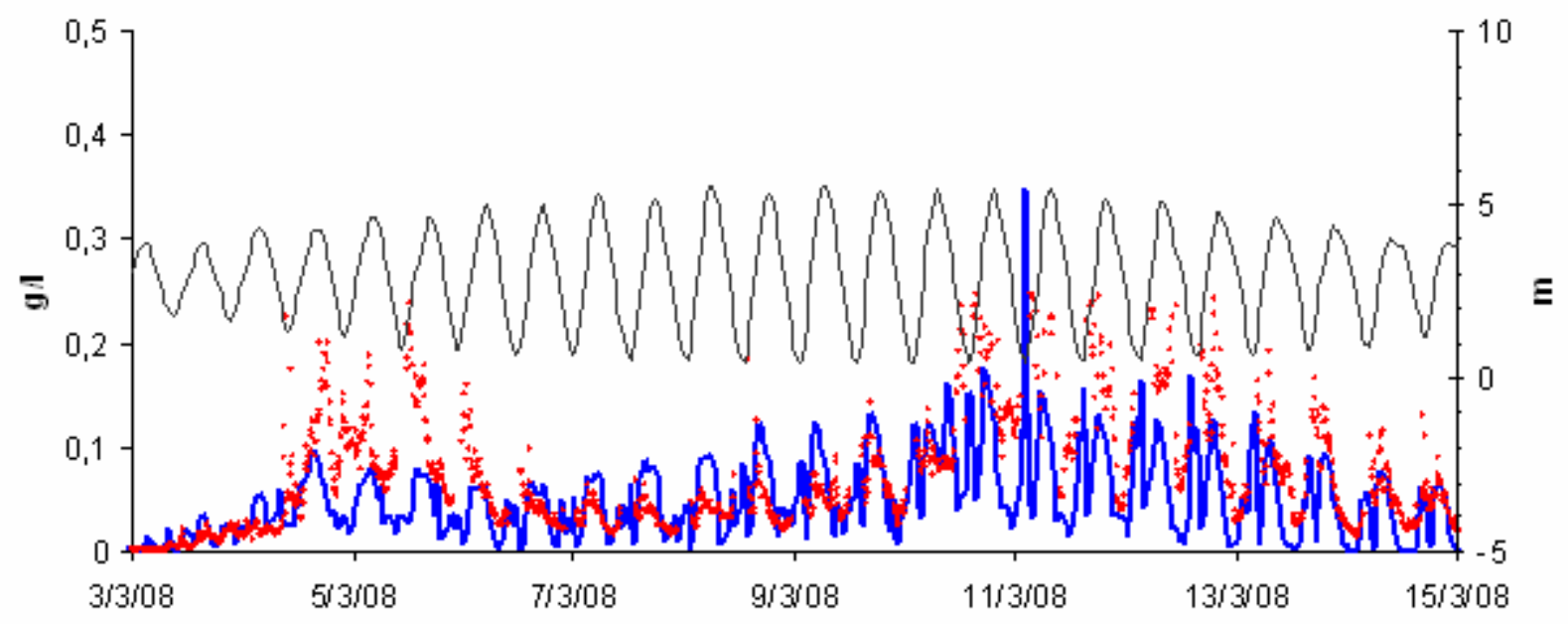

Figure 2 : Exemple de comparaison entre MES mesurée à $15 \mathrm{~cm}$ au-dessus du fond (points) et MES calculée par extrapolation des résultats du modèle $2 \mathrm{DH}$, en supposant un profil d'équilibre de Rouse (trait gras). Le signal de marée est représenté au-dessus. Résultats obtenus après un an de simulation.

\section{RESULTATS}

Par souci de concision, les résultats du modèle hydrodynamique ne sont pas présentés.

\section{V.1 Matières en suspension}

Comme le laisse comprendre la figure 2, la variabilité des turbidités dans le bassin de Marennes est très forte. La figure 3 en donne une illustration, pour le seul effet des courants de marée. On y observe un panache lié au bouchon vaseux de la Charente, et de fortes concentrations dans la lame d'eau qui envahit l'estran en flot. La dissymétrie entre l'Est et l'Ouest du bassin est très nette. Cette dissymétrie a aussi été constatée sur les salinités, indiquant le faible mélange transversal des masses d'eau, entretenu par le mouvement Nord-Sud de la marée et la résiduelle forte vers le Sud. 


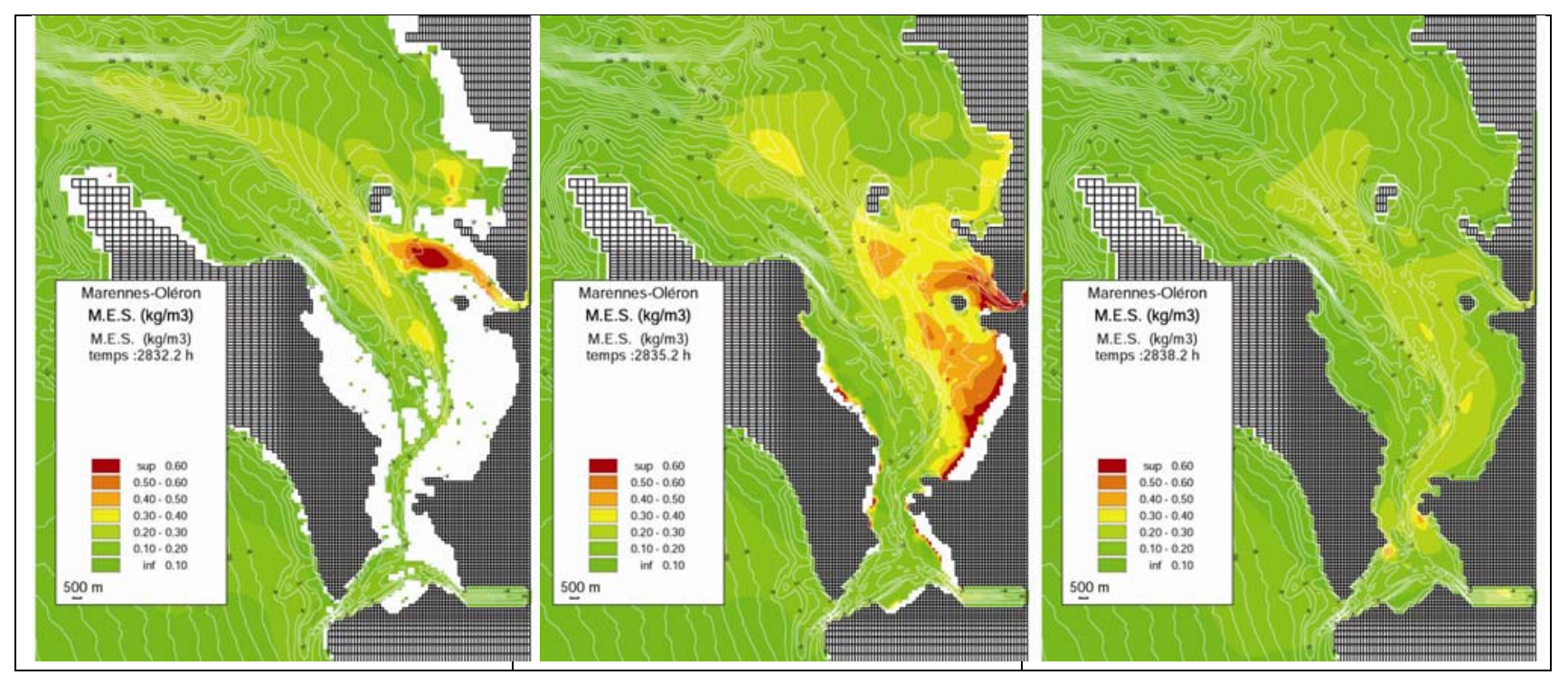

Figure 3: évolution des MES au cours d'une marée de vive eau: situations de basse mer (à gauche), de flot (au centre) et de pleine mer (à droite).

\section{V.2 Erosions/dépôts et flux}

L'examen des résultats du modèle pendant une année complète confirme l'importance des vagues (agitations locales et houle du large) dans la remise en suspension des sédiments. Cela se voit sur l'évolution de la masse totale de vase en suspension pendant 3 mois d'hiver, représentée sur la figure 4. La signature des seules vives eaux ne s'observe que de temps en temps, par exemple au cours des jours 300 ou 345 où les vents étaient faibles. La resuspension par les marées de vive eau s'élève à environ 1 million de tonnes, alors que le niveau de 2 millions de tonnes est dépassé 5 fois en 3 mois, sous l'effet des tempêtes d'hiver. Selon les résultats du modèle, la tempête du 9 décembre aurait provoqué la mise en suspension de 4 millions de tonnes. On peut noter que ces MES décantent assez vite, ce que confirment les observations (fig. 2).

En ce qui concerne les flux, on observe tout d'abord un transit des sédiments fins du Nord vers le Sud dans le bassin de Marennes, puisque les flux cumulés au niveau de l'île Madame (en rose) s'élèvent à 930000 t en 3 mois, orientés vers le sud, tandis que ceux calculés au Chapus (en bleu clair) sont de 500000 t, toujours vers le sud, et ceux transitant dans le pertuis de Maumusson (bleu foncé) sont évalués à 370000 t. « Au passage, on note un piégeage (donc un dépôt) de sédiments vaseux d'environ 400000 t dans le bassin Nord (au Nord du Chapus), et 100000 t dans le bassin Sud. Ces évaluations doivent être considérées avec prudence, car la robustesse de ces résultats en fonction des concentrations en MES à la limite marine ou des conditions d'érodabilité dans le Nord du bassin n'a pas été contrôlée. On relève aussi que ce flux vers le sud est essentiellement dû à la circulation résiduelle de marée, puisque les progressions sont clairement dominantes en vive eau. Au contraire, les tempêtes de décembre (SW pour la première, SW à NW pour la seconde) ont tendance à inverser les flux. Le flux à la Pallice, entre Ré et La Rochelle (en brun) est intéressant car il change de sens assez facilement. En particulier par tempête de SW il est orienté vers le nord, tandis que par vent de NW il s'inverse vers le sud. Le flux dans le pertuis d'Antioche est aussi remarquable : alors que sa valeur cumulée est faible, les flux instantanés au cours du flot et du jusant sont très élevés (oscillations de marée bien visibles sur la figure) : cela signifie que les échanges particulaires seraient équilibrés..., et que le flux très important arrivant dans le bassin par le nord proviendrait d'une érosion entre le pertuis d'Antioche et le continent. Cela est possible dans la modélisation grâce à une initialisation du sédiment en vase érodable à cet endroit, mais on peut se demander si le processus peut raisonnablement durer, car un fond vaseux n'est pas caractéristique d'une zone en érosion permanente. Une validation in situ semble nécessaire.

Enfin, on note que le flux résiduel à l'embouchure de la Charente dépasse 100000 t en 3 mois, et reste orienté vers l'amont. Ce résultat corrobore l'envasement constaté de l'estuaire de la Charente, alors que les apports amont sont faibles. II mériterait d'être confirmé par un calcul tridimensionnel reproduisant les circulations de densité à l'embouchure. 

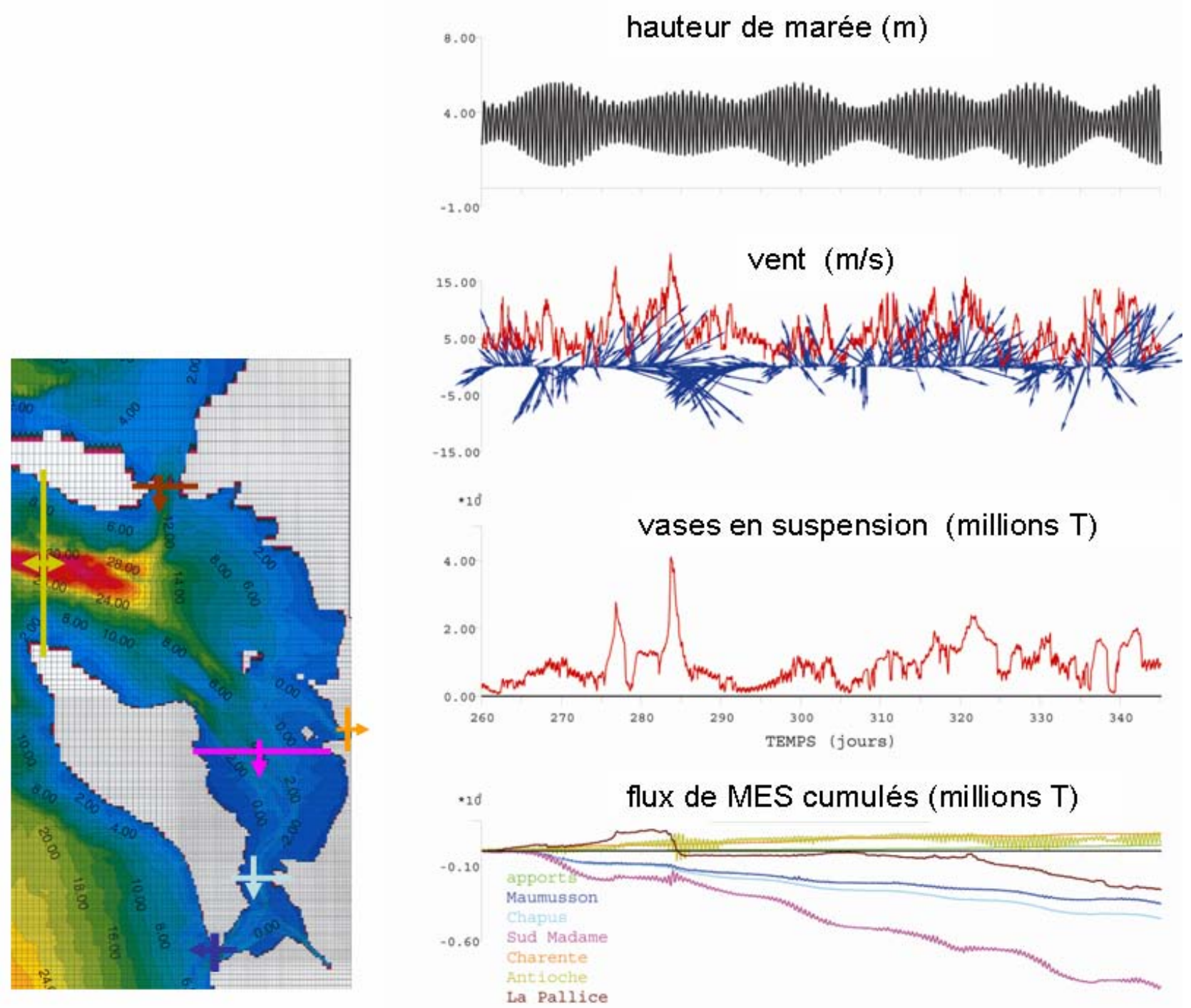

Figure 4 : Evolution du 15 nov.2007 au 8 fév.2008 de la masse de vases en suspension et des flux de particules fines à travers quelques sections du bassin (convention : flux positifs vers l'ouest ou le Nord)

\section{V.3 Variation de la nature du sédiment superficiel}

L'importance de la nature du sédiment superficiel pour la caractérisation des habitats benthiques a été rappelée en introduction. II est donc intéressant d'utiliser le modèle pour quantifier les possibles changements de nature du sédiment superficiel, souvent mal décrits par des observations trop peu fréquentes, et jamais transcrits sur les cartes. C'est ce qu'illustre la figure 5, en montrant que des dépôts (très superficiels) de vase peuvent se produire au cours des étales de vive eau (différence entre la situation de gauche et celle du centre), et en montrant qu'une tempête peut aussi modifier à la marge l'expansion des vases superficielles (différence entre la situation de droite et celle du centre). Ce type de résultat doit être analysé plus finement, en considérant aussi la structure verticale des dépôts de vases et les possibles laminations par des couches sableuses, ce qui s'observe sur les carottes numériques (le modèle multicouches le permet) comme sur les carottes in situ. 


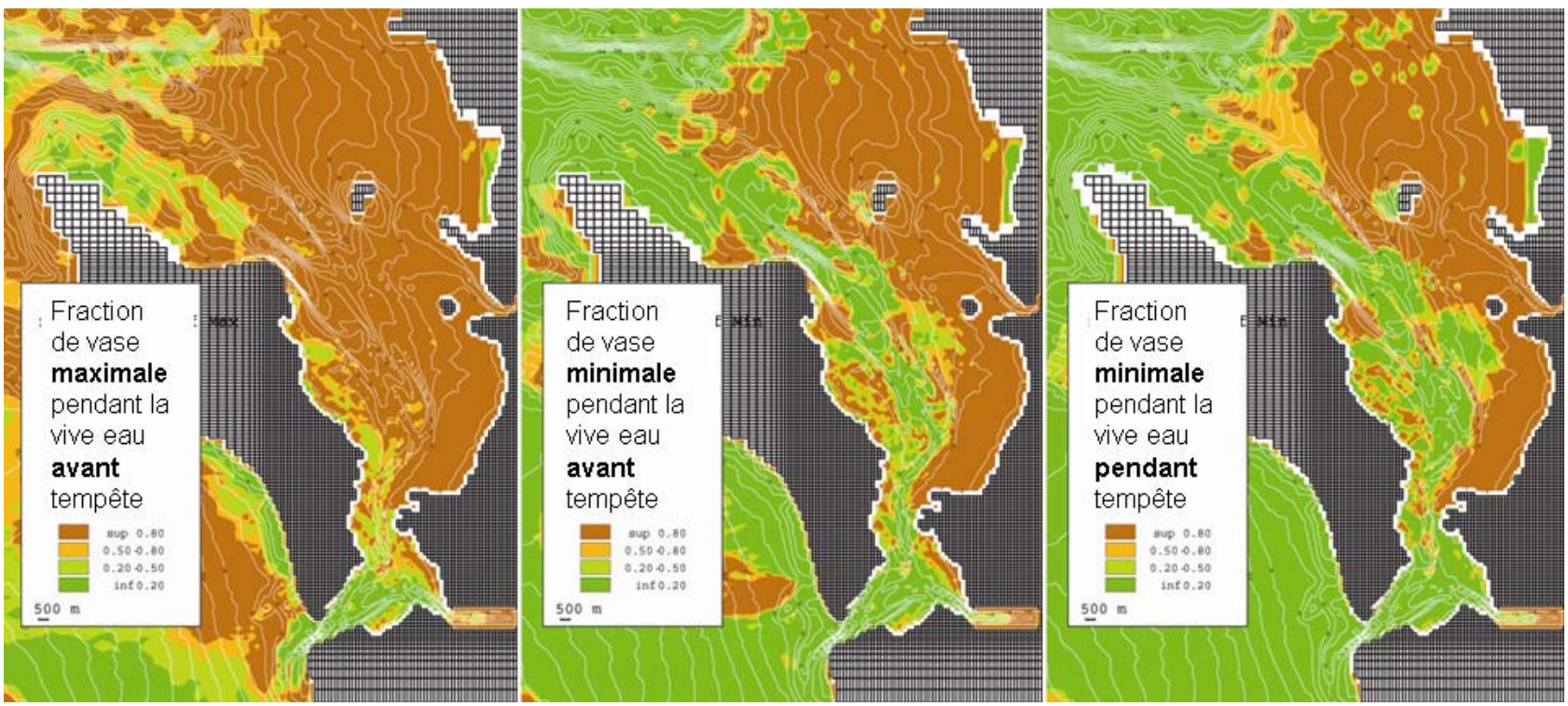

Figure 5: Variation de la fraction vaseuse dans le sédiment superficiel (2 premiers $\mathrm{mm}$ ), au cours d'une vive eau, et au cours d'une tempête.

\section{CONCLUSION}

Le modèle mis en place pour comprendre les processus sédimentaires dans le bassin de Marennes-Oléron rend compte des transports par les courants générés par la marée et le vent, et des remises en suspension par les vagues. Les sédiments cohésifs et non cohésifs ainsi que leurs mélanges sont considérés. La validation du modèle a bénéficié d'un ensemble de données de terrain exceptionnel. Le modèle s'avère très fiable pour restituer les circulations, tandis que la stratégie adoptée pour la simulation de l'effet des vagues, à partir d'une base de résultats de modèle pour des configuration types, n'est que moyennement satisfaisante, au regard des données disponibles: un calcul en mode instationnaire prenant en compte la chronologie du vent et des vagues au large devra être entrepris. La validation du modèle de transport sédimentaire est satisfaisante, mais elle a porté principalement sur les vases. II conviendrait de la poursuivre par une validation des flux sableux, plus difficile à concrétiser.

L'exposé rapide des résultats du modèle en termes de concentrations en MES, de flux particulaires et de leurs variations sous l'effet des tempêtes et des vives eaux donne l'impression que le modèle fournit les réponses (à l'échelle régionale) aux questions posées par les gestionnaires du milieu littoral (questions rappelées dans l'introduction). La robustesse de ces résultats doit cependant être vérifiée, en particulier vis-à-vis de l'initialisation 3D du sédiment dans le modèle. Par exemple, l'érosion de vase importante simulée entre le pertuis d'Antioche et la côte, qui contribue au flux d'entrée des vases dans le bassin de Marennes-Oléron, devrait être validée par des mesures altimétriques subtidales. Le modèle pourra être utilisé pour définir l'implantation optimale de tels capteurs. De même l'importance du flux d'entrée de MES dans le bassin mérite un suivi à long terme, que le modèle peut aider à optimiser.

Enfin, le modèle a permis d'illustrer la variabilité de la nature du sédiment superficiel, qu'il conviendrait de restituer dans les cartographies à venir de la couverture sédimentaire, indispensables pour la caractérisation des habitats benthiques.

\section{REMERCIEMENTS}

Le modèle hydrosédimentaire du bassin de Marennes-Oléron a été co-financé par le Conseil Général de Charente maritime et par Ifremer. Les calculs de propagation de vagues ont été réalisés par Creocean. La mise en œuvre des mesures de validation et leur utilisation pour la validation du modèle ont été soutenues par le Programme National en Environnement Côtier et dans le cadre du Contrat de Projet Etat - Région Poitou-Charente 2007-2013. La carte de nature du fond et les conditions harmoniques de marée au large ont été fournies par le Service Hydrographique et Océanographique de la Marine. Les données de forçage météorologique ont été acquises auprès de Météo-France dans le cadre du projet Previmer. Les auteurs remercient 
Serge Robert, Jean-Yves Stanisière, Romaric Verney et Hervé Jestin (IFREMER) et le personnel navigant de la station Ifremer de la Tremblade pour leurs contributions à l'acquisition et l'analyse des données in situ.

\section{REFERENCES}

[1] Bertin X., Chaumillon E. (2005). Apports de la modélisation sur bathymétries historiques dans la compréhension des évolutions des bancs de sable estuariens. C. R. Géosciences 337-15, 1375-1383.

[2] Brenon I., Le Hir P. (1999). Modelling the turbidity maximum in the Seine estuary (France) :identification od formation processes. Estuarine, Coastal \& Shelf Science 49, 525544.

[3] Le Roy R. \& Simon B. (2003). Réalisation et validation d'un modèle de marée en Manche et dans le Golfe de Gascogne. Rapport d'étude No002/03 du SHOM, 372EPSHOM/CH/GG/NP, 23 p.+annexes

[4] Creocean (2005). Etude pour une gestion dynamique des sédiments sur les côtes du bassin de Marennes-Oléron et le pertuis de Maumusson. Rapports du dossier 104213 pour le Conseil Général de Charente Maritime.

[5] Le Hir P., Waeles B., Cayocca F. (2009). Dynamics of sand and mud mixtures: a new modelling strategy. Continental Shelf Research, en révision.

[6] Weber N. et T. Garlan (2003). Les cartes sédimentologiques du SHOM des côtes VendéoCharentaises au 1/5000:7404 G et 7405G (des Sables-d'Olonne à l'embouchure de la Gironde), $9^{e}$ Congrès français de sédimentologie, livre des résumés vol. 38, ASF (2003), pp. 527-528

[7] Bassoullet P., Le Hir P., Gouleau D., Robert S. (2000). Sediment transport over an intertidalmudflat: field investigations and estimation of fluxes within the "Baie de Marennes-Oleron" (France). Continental Shelf Research 20, 1635-1653.

[8] Kervella S. (2009). Dynamique des sédiments cohésifs des zones intertidales centreatlantiques : cas de la baie de Marennes-Oléron. Caractérisation des sédiments, processus hydro-sédimentaires et modélisation appliquée. Thèse de doctorat, Université de la Rochelle

[9] Borsje B., de Vries M., de Boer G. (2008). Modeling large-scale cohesive sediment transport affected by small-scale biological activity. Estuarine Coastal \& Shelf Science 78, 468-480.

[10]Laboratoire d'Hydraulique de France (1995). Etude complémentaire des caractéristiques des vases dans le basin de Marennes-Oléron. Rapport C-0158-R1 pour Ifremer, 11p.+annexes. 